\title{
MENTAL CONDITION AND SPECIFICITY OF MENTAL DISORDERS IN A GROUP OF WORKERS FROM SOUTHERN POLAND: A RESEARCH REPORT
}

Jagiellonian University in Kraków, Kraków, Poland

Institute of Applied Psychology

\begin{abstract}
Background: The aim of this work is to provide empirical evidence regarding types and increasing prevalence of mental disorders affecting Polish working population in the years 2014-2016. The research questions concerned the specific characteristics of the types of mental disorders and their prevalence as well as the differences between males and females. Material and Methods: Types of mental disorders were investigated using a clinical method, a structured interview, as well as medical record data gathered in the years 2014-2016 in one mental health treatment center. The study was conducted in the population of 1578 working individuals aged 18-64 years old, in various forms of employment, including flexible employment (self-employment, task assignment agreement) and contract employment. The research population consisted of 998 females and 580 males, aged 18-64 years old. The study aimed at investigating types and the prevalence rate of mental disorders developed in the examined working Poles, also with reference to the sex of the study participants as well as the age at which they started seeking treatment. Results: The prevailing disorders include neurotic disorders; diagnosed according to the 10th Revision of the International Statistical Classification of Diseases and Related Health Problems (ICD-10) classification as a range of anxiety disorders, mixed anxiety-depressive disorders, stress-related and somatoform disorders; as well as personality disorders. The prevalence rate of the aforementioned disorders was found to be higher among working females than in the group of working males. Conclusions: The overall study conclusions based on the research data analysis point to the fact that the prevalence rate of various types of mental disorders displayed by the examined working males and females increased significantly in the years 2014-2016. Med Pr 2018;69(1):13-28
\end{abstract}

Key words: anxiety, mental health, mental disorders, mood disorders, working population, personality traits

Corresponding author: Bernadetta Izydorczyk, Jagiellonian University in Kraków, Institute of Applied Psychology, Łojasiewicza 4, 30-348 Kraków, Poland, e-mail: bernadetta.izydorczyk@uj.edu.pl

Received: June 17, 2017, accepted: August 17, 2017

\section{INTRODUCTION}

Recently, the subject of human mental condition and the quality of mental health in work situation is given increasingly more attention by researchers specializing in both psychology and medicine. The present-day deteriorating mental condition of working Poles is to some extent reflected in the growing spectrum of mental disorders observed in this population [1-5].

In the view of modern psychology and medicine, preventive healthcare ought to pertain to both physical (caring for one's own body) and mental health (caring for one's mental and social resources, and preventing mental disorders in a broad sense). Prevention and promotion of mental health is a task and an objective each person pursues in order to become able to satisfy one's psychosocial needs, among others. Outside the family and the social context, psychosocial needs (e.g., security, self-actualization, emotional closeness, social contact) are met also by means of functioning in a satisfying working environment. The deteriorating mental condition of Poles, including those economically active, is suggested by the statistics and reflected in the upward trend documented for the incidence rates for various mental disorders identified in the Polish population.

One of the mental disorders particularly often reported by modern Poles are the so-called neurotic disorders manifested in various forms of anxiety states and various psychogenic somatic ailments [3-5]. Emotional crises and emotional disorders constitute a common element of neurotic reactions and disorders occurring in the working population. The article presents an attempt at determining empirically the severity of the upward trend both in the incidence and in the range of mental disorders in the working Polish population. The interest in the subject also stems from the possible impact exerted by the increased incidence of mental disorders amongst the workforce on the functioning of economically active people over their lives after diagnosis while fulfilling multifaceted personal, familial and occupational functions. 
A worker who experiences mental disorders finds him- or herself in a situation that is difficult on numerous levels and consequently hinders the fulfilment of psychosocial needs, including those professional and familial. As a result of the experienced long-lasting symptoms of mental disorders and emotional difficulties, such a person limits his or her own ability to execute occupational tasks, leading to the development of secondary psychopathology of a given disorder and over time restricts the worker's positive attitude to his or her professional activity, limiting his or her positive attitude towards a given professional activity and occupational tasks. The above-described situation results in frequent sickness absenteeism and medical treatment that oftentimes requires rehabilitation benefits or pensions to be provided for the suffering employees. Mental disorders experienced by workers often pertain to their emotional experiences and personality.

The specificity of symptoms and the course of emotional disorders usually require employees to seek treatment in outpatient centers without the need to resort to sick leaves. If treatment and psychological assistance are not provided in time, the symptoms of emotional disorders (oftentimes anxiety, depression, psychosomatic symptoms) usually become more prevalent and persistent, compromising the physical and mental condition of the worker, resulting in him or her suffering from occupational exclusion, confining his or her role to the one of an 'ill' person or even strengthening that role. In conclusion, the quality of mental health and the prevention of mental disorders in the workforce is a significant issue both for the employee and the employer, since in both cases it entails negative consequences reflected in a compromised ability to fulfil the needs and tasks related to the occupational function.

Clinical psychology and psychiatry contribute to industrial and organizational psychology by introducing research findings and theories in the field of the development of psychopathology of mental disorders, allowing a person in the context of work to be seen from many different diverse perspectives. One of these perspectives indicates the need to take psychological resources of economically active people into consideration in occupational psychology when it comes to the application of preventive healthcare (caring for one's own physical and mental health). Another perspective indicates that occupational psychology needs to take into consideration the impact of various mental disorders that have become increasingly common for the workforce [5-7].
Mental disorders amongst economically active persons may develop due to factors related to work situation (e.g., emotional crises caused by burnout or mobbing) or when performing occupational functions regardless of the cause (e.g., depression, neurotic disorders, stress-related disorders, psychosomatic disorders, personality disorders).

On the one hand, clinical psychology indicates directions in preventive healthcare that aim at promoting mental health among individuals in work situation. On the other hand, owing to the background knowledge in the field of health psychology, stress psychology and psychopathology we become aware of the sort of psychological dysfunctions and mental disorders that may accompany a person fulfilling his or her occupational function. Professional work is a major activity in human life and is as important as playing in childhood. An individual concurrently serves many social (a member of numerous social circles) and personal (family) roles in work situation. For that very reason, various mental disorders experienced by that person have a wideranging destructive impact on his or her functioning in both social and professional groups, covering many areas of existence including family life, social life and professional life.

According to the definition developed by the World Health Organization (WHO) [29] sound health requires complete bio-psycho-social balance and not merely an absence of disease. Fulfilling one's occupational function on a daily basis requires from that person to preserve both physical and mental health. Ensuring good mental health in work situation constitutes a major element of one's proper functioning as a worker, i.e., a function that significantly improves security. Economic activity fosters fulfilment of numerous needs (self-actualization, respect, positive regard and affiliation, belongingness), which promotes good mental health, yet may also have an impact on the recovery.

Therefore, promotion of mental health in work situation requires combining efforts taken by the worker him- or herself and the social environment (family, the employer, other environmental circles). Ensuring healthcare in the context of work constitutes an area of labor law that should be of interest to every employer, as it imposes an obligation to observe many orders and prohibitions arising from laws and regulations, but also to the employee. The right to hygienic and safe working conditions is guaranteed by the constitutional rights and liberties whereas in the context of labor law it has been upgraded into a basic provision of labor law. In many 
states, psychosocial and environmental factors at the workplace combined with the associated stress have been considered a significant threat to employees' health. In the European Union, the most important legal act pertaining to this issue is Directive 89/391/EEC on the introduction of measures to encourage improvements in the safety and health of workers at work [1].

According to the WHO, mental diseases will have become one of the major health problems in the European population by 2020 [2]. Results of studies conducted in Europe and published in 2011 as a report suggest that each year 164.8 million European citizens (38.2\%) suffer from mental disorders $[3,4]$.

In the Polish epidemiological study on mental disorders named "Epidemiology of mental disorders and access to mental health care" ("Epidemiologia zaburzeń psychiatrycznych i dostępność psychiatrycznej opieki zdrowotnej" - EZOP), which was conducted in 2012 on a population of 10000 people aged 18-64 years old, $23.4 \%$ participants proved to have experienced at least 1 mental disorder in their lifetime [5]. The high incidence of mental disorders in the adult Polish population with regard to sex and age is on the rise. Hence, the need for psychiatric and psychological healthcare is also growing. Apart from the growing incidence of disorders caused by alcohol abuse, EZOP also reports an upward trend in the incidence of non-psychotic disorders (neuroses and personality disorders) in the adult Polish population. The group of people with neuroses and personality disorders also involves economically active individuals. According to the study results, about $20-30 \%$ working-age Poles reported symptoms of mental disorders classified as: neurotic, stress-related and somatoform disorders, mood disorders and personality disorders, as well as psychoactive substance use disorders. The most common non-psychotic disorders are anxiety disorders $-10 \%$ of the study population (i.e., about 2.5 million persons) and depressive disorders [5].

In Poland, the subject of empirically verified distribution of various mental disorders in the working population is seldom discussed in scientific research. Still, if discussed, it pertains most often to studies on the role of mentally ill people in the labor market, as examined in the studies conducted by Wciórka and Wciórka [3] or Kaszyński and Cechnicki [4,5]. Worldwide literature and research topics it elaborates on refer to various aspects of the functioning of the following individuals:

diagnosed with schizophrenia and their occupational function, e.g., research by Mallick et al. [8],
Bond et al. [9], Koletsi et al. [10], Bio and Cottaz [11], Kiviniemi et al. [12], McGurk and Maltzer [13],

diagnosed with depression in work situation, e.g., research by Gilmore and Palten [14], Lerner et al. [15], Burton et al. [16], Backenstrass et al. [17], Beck et al. [18], Wang et al. [19].

The literary analysis indicates that there are no research reports describing emotional disorders (i.e., neuroses and personality disorders) present in the working population apart from mental disorders, although these groups of disorders are diagnosed far more often in the adult Polish population of both sexes [5]. For that very reason, undertaking the research topic aiming at providing an empirical verification of the specificity of emotional disorders in economically active individuals may significantly contribute to broadening of the source material with regard to the clinical aspects of industrial and organizational psychology.

In clinical psychology and medicine, basic types of emotional disorders are defined as neuroses, stressrelated disorders, somatoform disorders or personality disorders. A person may experience each of the aforementioned types of disorders throughout his or her professional activity. A stressful situation may contribute to the emergence of various types of mental disorders, from emotional crises to neuroses or personality disorders.

In contrast to mentally ill individuals described in the literature, in the context of difficulties encountered by these individuals in accessing work and performing professional tasks, many people with neuroses and personality disorders most often remain in the workforce. In the case of people with neuroses and personality disorders, functioning in stressful working environments usually contributes to an intensification of various emotional and social difficulties, which hinders fulfilment of their occupational roles. Clinical aspects of industrial and organizational psychology (stress psychology, crisis psychology and psychopathology of disorders) constitute a significant factor that stimulates the process of promoting mental health and supporting workers in their search for potential sources of stress and preventing chronic stress at work.

Mental healthcare pertains to caring for an individual and his or her health not solely in the context of work. The Mental Health Act adopted in 1994 [20-22] with the aim to protect the rules and principles of conduct in relation to persons with mental disorders and the National Mental Health Protection Program [21,22] adopted by the Council of Ministers in 2010 determine 
the basic standards of conduct in the prevention and treatment of people with mental disorders and various emotional difficulties. In "Health 2020. A European policy framework and strategy for the 21st century", the World Health Organization indicates that good health is essential for economic and social growth [21,22]. The above-mentioned document draws attention to the necessity to ensure proper national strategic framework for executing key thematic priorities concerned with healthcare also in the working population. Furthermore, apart from factors related to family life, the implications of mental disorders and emotional crises occurring in one's life are also related to his or her work situation.

Another vital element that disturbs the functioning of a mentally sound individual is the phenomenon called occupational stress. It usually stems from work situation, yet a developing emotional disorder or neurotic symptoms may constitute a significant contributing factor that stimulates the formation of a stressful situation also in the context of work. In psychology and medicine (particularly in psychiatry), theories of stress explain the origin and the mechanisms for the development of emotional crises whereas theories of psychopathology explain the origin and the formation of the mechanisms underlying mental disorders. The leading theoretical concepts in the literature that provide a scientific explanation for the phenomenon of stress provide for the transactional model of stress and coping by Lazarus and Folkman [23], Hobfoll's conservation of resources theory [24], and Maslach and Leiter's theory [25,26].

The implications of a stressful situation are incurred not solely by the worker but also by his or her co-workers and family [27,28]. Scientific concepts of stress indicate that a significant role in the occurrence of stress is played by a threat and a loss that may result from work situation [28]. The state of frustration due to unmet psychosocial needs may result in chronic stress that manifests itself as the following symptoms: anxiety, aggression, depression and somatic symptoms. Stress at work in itself may trigger the worker's reaction in the form of an emotional crisis, yet it may also be the source of the following symptoms: anxiety, depression, insecurity, aggression and psychosomatic symptoms.

The worker may also suffer from various mental disorders (regardless of the sources related to work): neuroses, personality disorders, psychotic disorders. The very specificity of symptoms of these disorders may have an adverse effect on the course of adaptation and execution of occupational tasks if the person concerned does not seek treatment. A determined distribution of the types of mental disorders amongst employees should contribute to providing them with the necessary treatment and support in their recovery.

Considering the above presented theoretical premises of the research topic, the primary objective of the study was to perform an empirical verification of types of mental disorders prevalent in the group of economically active Poles. The incidence and the distribution of mental disorder types determined based on medical diagnoses in the study population issued in the years 2014-2016 were subject to clinical and statistical analyses. In line with the research assumptions, the pursued objective was to define the incidence and the distribution of the types of the so-called non-psychotic disorders, i.e., neuroses and personality disorders. The other objective of this study was to make an attempt to determine the following socio-demographic characteristics in the economically active participants: age, sex and the age at first treatment sought by the working individuals due to the experienced mental disorders [29].

All procedures performed in studies involving human participants didn't violate the Personal Data Protection Act and were in accordance with the ethical standards of the institutional research committee and with the 1964 Helsinki declaration and its later amendments or comparable ethical standards. Ethical approval was granted by the Ethics Committee of the Institute of Applied Psychology of the Jagiellonian University in Kraków. Informed consent was obtained from all individual participants included in the study.

The key research questions are:

1. Did the incidence with respect to the types of mental disorders as classified in 10th Revision of the International Statistical Classification of Diseases and Related Health Problems (ICD-10) grow notably in the working population in the years 2014-2016?

2. What is the distribution of the incidence and the type of mental disorders in the study population of economically active individuals in 2014-2016 and what is it characterized by?

3. Is there any significant difference between the female and male participants with regard to the incidence and the presented types of mental disorders, and if so, where does it lie in?

The following variables were included in the design of the study:

Mental disorders - a variable that covers a set of various mental disorders specified in medical records in a nosological diagnosis, determining the level of 
absence of mental health in an individual who is employed and executes employment-related tasks. This variable was characterized by means of specific nosological diagnoses of mental disorders made by a psychiatrist for a study participant. The nosological diagnosis of the type of mental disorder was made due to the International Statistical Classification of Diseases and Related Health Problems [30]. Each medical diagnosis was made by the same permanent team of 4 collaborating psychiatrists with at least 15 years of professional experience, who were all professionally active (conducting research) as a team in the years when participants were sampled for the study. They were all holders of specialization degrees in psychiatry who made medical diagnoses based on ICD-10 and criteria common for all diagnosticians provided in the structured clinical interview. The interview criteria pertained to the measurement of clinical history and autobiographical data focused on diagnosing the symptomatology of the reported disorders. The study population involved individuals with the following diagnostic categories (due to ICD-10): personality disorders (F60), eating disorders (F50), neurotic, stressrelated and somatoform disorders (F40-F48), mood disorders (F30-F34), schizophrenia, schizotypal and delusional disorders (F20-F29).

Professional activity - a variable that involves active participation of a subject in work situation for at least a year. The said subject had been or was functioning as an employee. This variable was characterized by means of an actual ongoing (for $>7$ months at least) participation in work situation (fixed-term contract of employment, part-time contract of employment, business activity, ongoing seasonal employment, temporary employment). The variable was measured based on relevant data derived from a clinical interview and a medical record.

\section{MATERIAL AND METHODS}

The research variables were measured using a method that was recognized in the field of clinical studies, namely, a structured interview, and the following data from medical records: an observation chart with information on the medical history and socio-demographic data that allowed the characteristics of the research variables to be determined (paragraphs 1 and 2). The observation chart in the medical records of the study participants included data on the following characteristics:
1. A nosological diagnosis of the type of mental disorder due to ICD-10: anxiety disorder, mixed anxiety and depressive disorder (F40-F42); dissociative disorder, somatoform disorders, stress-related disorder (F43); somatoform disorders (F44, F45, F48); eating disorder (F50); specific personality disorders (F60); other mental disorders (affective disorder F30 and schizotypal disorder F20).

2. Socio-demographic characteristics: age, sex.

3. Data pertaining to the beginning of treatment due to mental disorders.

In the research procedure, records of the above listed characteristics of research variables were employed, collected in the years 2014-2016 in a mental health clinic. The research questions and the verified research variables did not require any measurements with the use of psychological tests. Determining the type of mental disorder using a psychological test was not necessary because of the medical diagnosis provided a sufficiently precise diagnostic rate of the type of mental disorder to be employed in the study. There was no need to employ another strict measurement of rates aiming at identifying the type of a given mental disorder. The documented medical diagnoses made by a psychiatrist due to ICD-10 and the necessity of the study participants to seek treatment served as an adequate characteristic for measuring the mental disorders variable.

\section{Participants}

Due to the set research objectives, the study included a targeted selection of participants. The target population comprised 1578 individuals aged 18-64 years old who remained economically active and held various forms of employment at the time of the study ranging from the so-called flexible forms of work organization (self-employment, task assignment agreement) to contract employment. The study group involved 998 women and 580 men aged 18-65 years old (mean age of the total population $=35.4$ years old). The mean age was 35.4 years old for female participants and 39.4 years old for male participants.

The study included a group of women and men who were economically active at the time of the study, and at the very same time had a record of mental disorders medically diagnosed as emotional disorders. This group of disorders is often specified as part of the spectrum covered by the treatment tasks in the so-called minor psychiatry. These most often include: neuroses in a broad sense, stress-related disorders, personality disorders, somatoform disorders, as well as eating 
disorders, affective disorders and schizotypal disorders. Given the nature of symptoms and the specificity of treatment, the group of people with the above described types of disorders is most often admitted to Mental Health Outpatient Clinics and Centers for Anxiety Disorders. The study involved economically active persons who sought medical psychiatric help and received counselling services in a Mental Health Outpatient Clinic or a Centre for Anxiety Disorders at the same time.

\section{Design and procedure}

In order to obtain the research material and the answer to the research questions, the study participants were selected to the group by means of analyzing the data collected from the economically active patients who reported to the Mental Health Outpatient Clinic and the Centre for Anxiety Disorders in the years 2014-2016. Importantly, the 2 facilities selected for the study were operating as part of a larger center for neurotic and personality disorders in southern Poland, that concluded a long-term contract with the National Health Fund and rendered healthcare services throughout Poland. Due to its metropolitan environment, the said medical center provided wide access to various occupational groups that were using services rendered by psychiatrists and psychologists.

The objective of the study did not cover the need to analyze the distribution of education in the study population of economically active Poles diagnosed with mental disorders. The research procedure involved only collecting clinical data over 3 consecutive years using a percentage distribution of the quantity of the study participants in terms of the measurement of the incidence and the type of mental disorders in the study population. In 2017, all the obtained research data was subject to a summary analysis.

\section{RESULTS}

In order to answer the research question, the following statistical analyses were performed. Answering the first, the second and the third research question required primarily computing a series of descriptive statistics provided in the Table 1 and in the Figures 1-4. Next, a statistical analysis was carried out to specify the study population regarding age at which the treatment was commenced due to mental disorder symptoms experienced by the participants according to the records (Figure 5).

Table 1. Mental disorders among the economically active respondents in Poland, 2014-2016*

\begin{tabular}{|c|c|c|c|c|c|c|}
\hline \multicolumn{7}{|l|}{2014} \\
\hline females [n] & 200 & 315 & 30 & 0 & 27 & 13 \\
\hline males [n] & 160 & 144 & 1 & 2 & 16 & 10 \\
\hline \multicolumn{7}{|l|}{2015} \\
\hline females [n] & 198 & 268 & 73 & 24 & 35 & 13 \\
\hline males [n] & 170 & 103 & 8 & 24 & 16 & 9 \\
\hline total [n (\%)] & $368(23.32)$ & $371(23.51)$ & $81(5.13)$ & $48(3.04)$ & $51(3.23)$ & $22(1.39)$ \\
\hline total $[\mathrm{n}(\%)]$ & $850(53.86)$ & $864(54.75)$ & $132(8.36)$ & $169(10.70)$ & $10(0.63)$ & $0(0.00)$ \\
\hline Total [n] & 1578 & 1694 & 244 & 219 & 104 & 45 \\
\hline
\end{tabular}

* The percentage distribution of specific types of mental disorders due to 10th Revision of the International Statistical Classification of Diseases and Related Health Problems (ICD-10) was compared to the total number of all mental disorders in 2014-2016.

a Some respondents have both primary and subsidiary medical diagnosis (F40-F48), e.g., primary F40 and subsidiary F41.0.

F40-F48 - neurotic, stress-related and somatoform disorders, F50 - eating disorders, F60 - specific personality disorders, F30-F34 - affective disorders,

F20-F29 - schizophrenia, schizotypal and delusional disorders. 


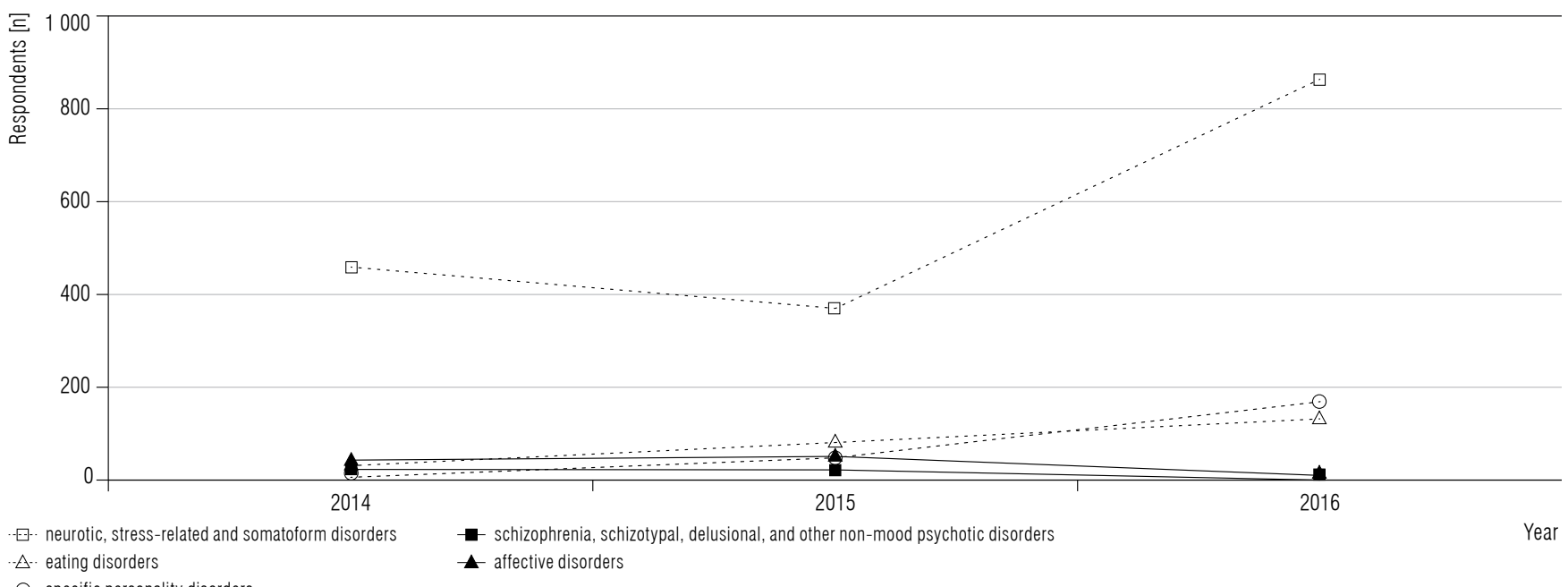

$-A$. eating disorders

- affective disorders

- $\odot$. specific personality disorders

Statistically significant differences estimated using a $\mathrm{Chi}^{2}$ test $\left(\mathrm{Chi}^{2}(8)=221.89, \mathrm{p}<0.001\right)$.

Fig. 1. Mental disorders among the economically active respondents $(\mathrm{N}=1578)$ in Poland, 2014-2016

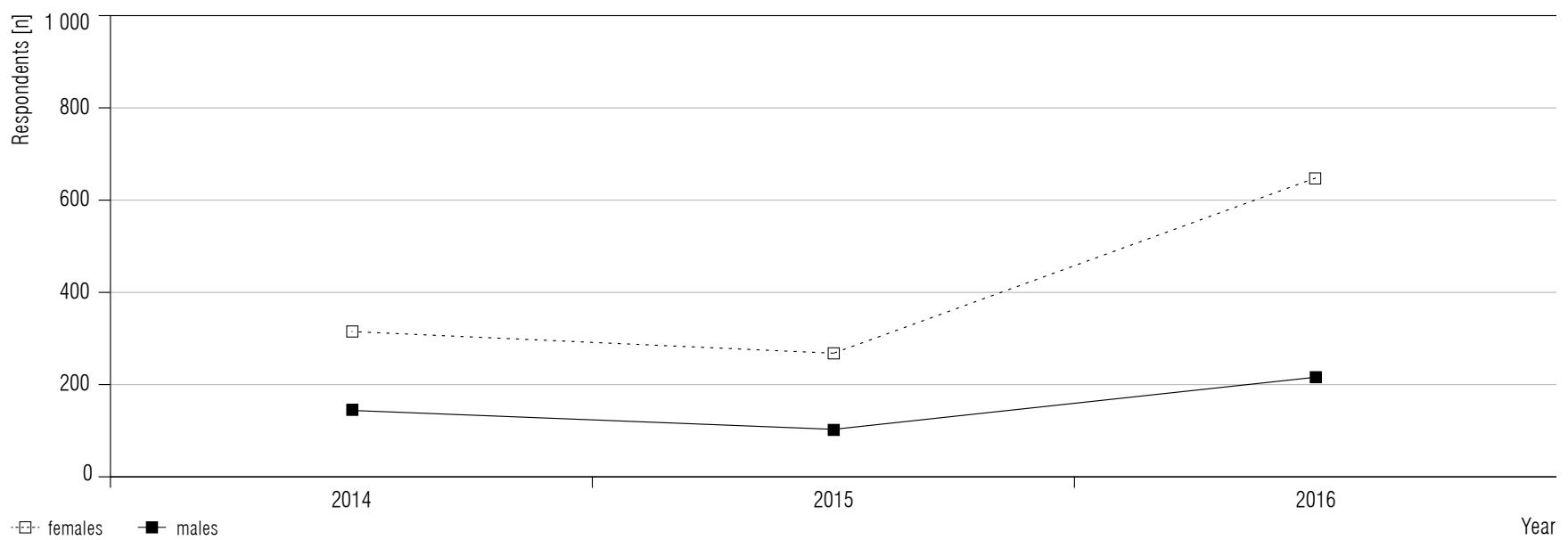

Statistically significant differences estimated using a Chi ${ }^{2}$ test $\left(\mathrm{Chi}^{2}(8)=221.89, \mathrm{p}<0.001\right)$.

Fig. 2. Neurotic, stress-related and somatoform disorders among the economically active females $(\mathrm{N}=998)$ and males $(\mathrm{N}=580)$ in Poland, 2014-2016, by sex

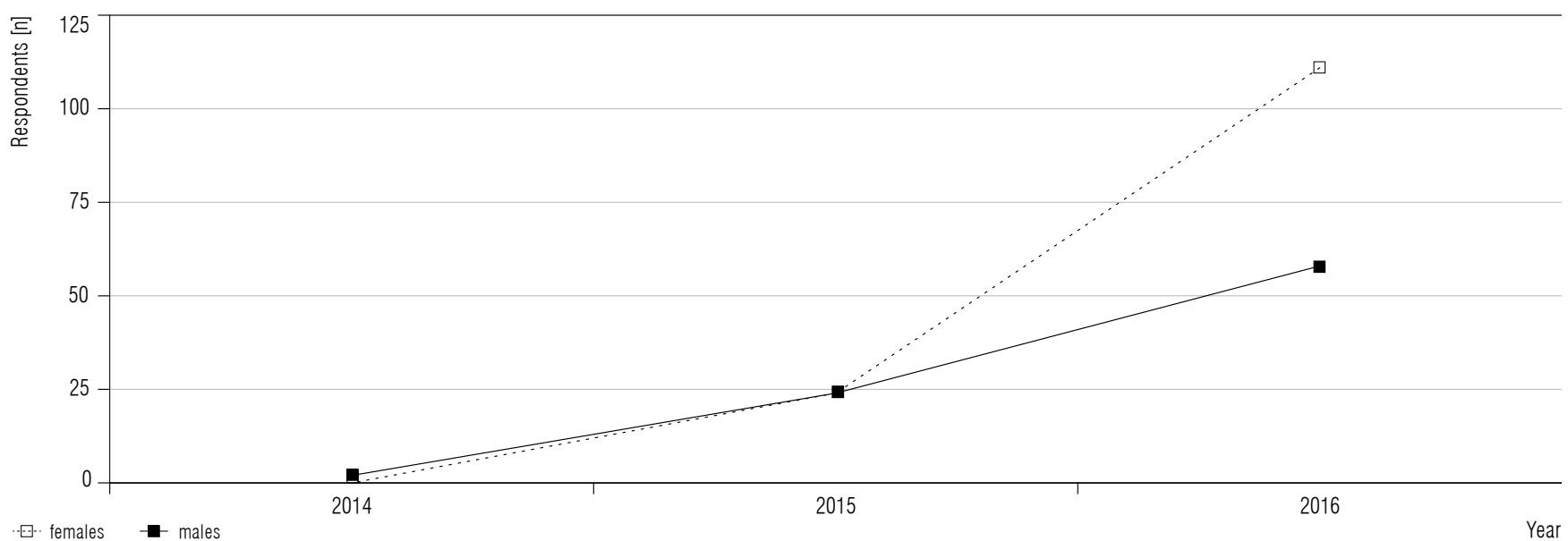

Statistically significant differences estimated using a Chi ${ }^{2}$ test $\left(\mathrm{Chi}^{2}(8)=221.89, \mathrm{p}<0.001\right)$.

Fig. 3. Specific personality disorders among the economically active females $(\mathrm{N}=998)$ and males $(\mathrm{N}=580)$ in Poland, 2014-2016, by sex 


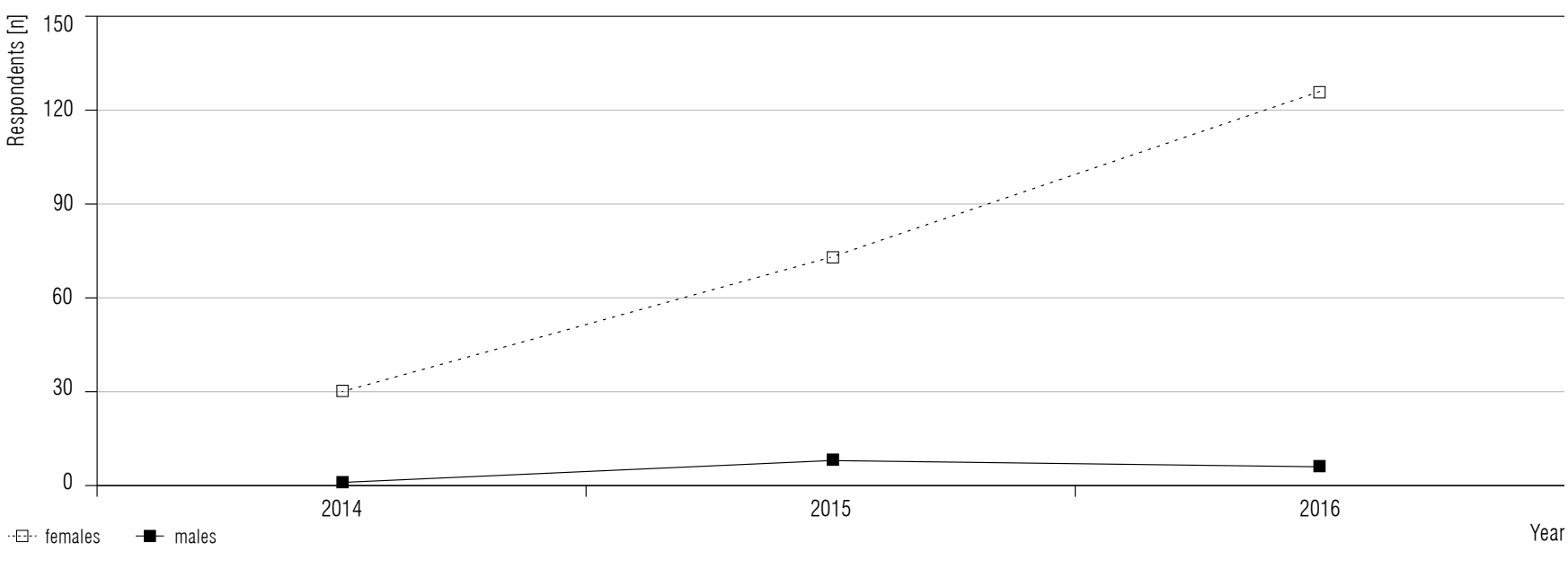

Statistically significant differences estimated using a Chi ${ }^{2}$ test $\left(\mathrm{Chi}^{2}(8)=221.89, \mathrm{p}<0.001\right)$.

Fig. 4. Eating disorders among the economically active females $(\mathrm{N}=998)$ and males $(\mathrm{N}=580)$ in Poland, 2014-2016, by sex

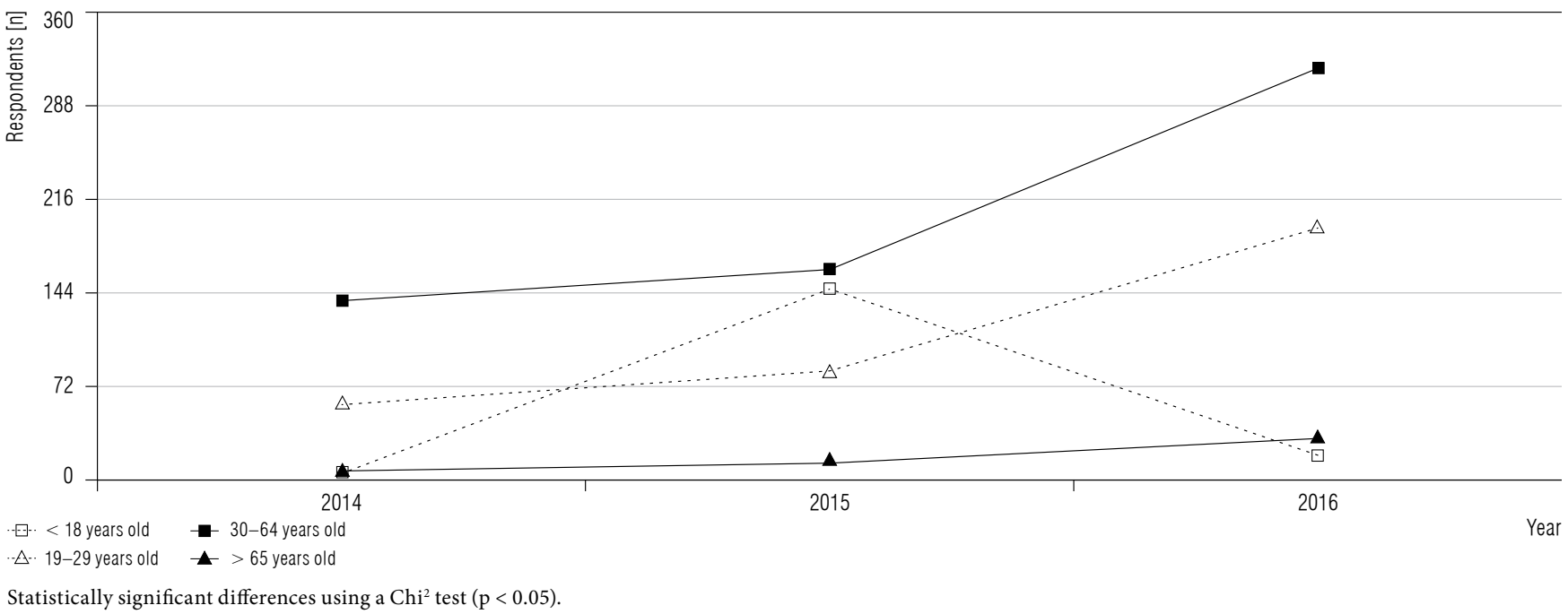

Fig. 5. Age at first treatment due to mental disorders among the economically active respondents $(\mathrm{N}=1578)$ in Poland, 2014-2016

The characterization of the mental disorders variable with regard to the distribution of the incidence of various types of mental disorders in the study population in the years 2014-2016 was carried out by means of measuring the number of participants with specific types of mental disorders verified and documented in the medical records. As mentioned above, when the variables were defined, the following main diagnostic categories specified in ICD-10 were subject to the analysis. These are: anxiety disorder, mixed anxiety and depressive disorder (F40-F42); dissociative disorder, somatoform disorders, stress-related disorder (F43); somatoform disorders (F44, F45, F48); eating disorder (F50); specific personality disorders (F60); other mental disorders (affective disorder F30 and schizotypal disorder F20).

Next, the female participants were compared with the male participants with regard to the specificity of the distribution of the type and the incidence of mental disorders in the study population in the years 2014-2016. In the last stage of the data analysis, the study group was characterized in terms of socio-demographic data, i.e., sex, age, place of residence, education, work experience and the type of work performed.

The study results were subject to the statistical analysis using SPSS 19 software. Variations of selected categories of qualitative variables were determined by means of a $\mathrm{Chi}^{2}$ test. The statistical significance was estimated at $\mathrm{p}<0.05$. In order to clarify the obtained data, only statistically significant results are shown in the Figures 1-5.

In the first stage of the statistical analysis of the study results descriptive statistics were measured the quantity of the mental disorders variable. On this basis, the statistical significance of differences in the 
incidence and the distribution of mental disorders in the years 2014-2016 was determined first for the entire study group (Table 1) and then for the groups of male and female participants separately (Figures 2-4).

In the second stage of the statistical analysis, the distribution of the study results describing socio-demographic characteristics was analyzed for the entire study population and for the groups of male and female participants separately (Figure 5).

\section{Clinical and statistical analyses of the incidence of mental disorders in the groups of economically active participants}

The results of the study determining the overall incidence of various types of mental disorders in the entire study population of individuals who were economically active in the years 2014-2016 are presented in the Table 1 and Figure 1 whereas the Figure 2 shows the incidence of mental disorders for the groups of male and female participants separately.

The results were analyzed first by measuring the percentage distribution of mental disorders (according to medical diagnoses due to ICD-10). This measurement was made with reference to the total number of medical diagnoses of all the specified mental disorders in consecutive years 2014-2016. This aimed at verifying whether the incidence of disorders in the study population increased in the years 2014-2016 and determining the growth rate. The incidence of medical diagnoses in the study population was analyzed due to ICD-10 classification. All psychiatrists were expected to adjust their clinical interviews to the same criteria supporting medical diagnoses due to ICD-10 (autobiographical data of the patient that supports source data concerning symptoms).

The study results regarding the incidence of all mental disorders suggest a significant growth in the number of economically active individuals who sought help due to various mental disorders in the years 2014-2016 at the very same health facilities where the study measurements were conducted. In all cases, the highest percentage of people who sought medical help in 2014-2016 was represented by working individuals with neuroses. Based on the percentage distribution of the frequency, $54 \%$ of all the study participants were diagnosed with neuroses in 2016.

Economically active individuals diagnosed with disorders classified as ICD-10: F40-F48 accounted for 29\% in 2014 and for 23\% in 2015. The second largest group manifesting mental disorders were economically active persons diagnosed with various types of person- ality disorders. However, in this case the percentage of reported cases was considerably lower in comparison to neuroses, with the number of cases reported in 2016 being the highest (slightly over 10\%), far lower in 2015 (5\%) and marginal in 2014 (0.12\%).

As for eating disorders (anorexia, bulimia, binge eating disorder and other disorders classified as F50), over $8 \%$ in 2016, over 5\% in 2015 and nearly $2 \%$ in 2014 of the total study population were manifesting eating disorders. The least common categories in terms of incidence in the study population who reported to the facility where the study was carried out were affective disorders (F30-F34) and schizophrenia, schizotypal and delusional disorders (F20-F29).

The above-presented distribution of diagnosed mental disorders may be related to the specificity of the study group, which comprised patients reporting to the selected Mental Health Outpatient Clinic and the Centre for Anxiety Disorders, the specific services of which included psychotherapy and to an extent limited the access to this form of treatment for patients with mental disorders in cases of severe depressive (episodes of mild and severe depression) and psychotic (psychoses) symptom decompensation. There were no drug and alcohol addicts in the study group. Since the specified group of economically active individuals is usually referred to inpatient psychiatric facilities, those classified to this subgroup were not referred to outpatient treatment and did not take part in the analyses conducted as part of this study. In this respect, the mentioned limitation pertaining to the sampling is worth a more detailed examination in further research.

In conclusion, the obtained study results provided in the Table 1 and the Figure 1 suggest a significant growth in the number of economically active individuals reporting to the Mental Health Outpatient Clinic and the Centre for Anxiety Disorders due to experienced mental crises and various types of mental disorders (particularly neuroses and personality disorders) in the years 2014-2016. On the one hand, this might imply an actual growth in the incidence of mental disorders and a deteriorating mental condition in working Poles. On the other hand, the data might suggest that economically active Poles seek medical and psychological help more often due to experienced mental difficulties and psychopathological symptoms. The growing trend in mental disorders in the study population of economically active individuals in the years 2014-2016 is presented in the Figure 1.

The statistical analysis of the measurement results for the average incidence of specific types of mental 
disorders in the study population provided answers to the 2 first research questions. The research data shown in the Figure 1 confirms the significant growth in economically active individuals who reported various mental disorders in the years 2014-2016. The statistical significance of differences in all types of comparisons was high $(\mathrm{p}<0.001)$. The highest growth $(2$-fold higher since 2014) was observed in the number of people diagnosed with neurotic, stress-related and somatoform disorders. A statistically significant increase (though weaker than in the case of neuroses) pertains to people diagnosed with various types of personality disorders.

A weaker growth compared to 2014 was noted for eating disorders. This situation may be explained to some extent by the fact that eating disorders constitute a group of disorders that affect mainly women who are not eager to seek medical and psychological help. In the male population, this type of disorder was noted to be far less common compared to the incidence noted for the female participants. Nevertheless, the mean age of persons diagnosed with mental disorders is slightly over 35 years old for women and 39 years old for men, which is inconsistent with the statistical data that indicates the prevalence of eating disorders in a notably younger female population (adolescents and young adults).

The smallest variations in the incidence in the years 2014-2016 were observed for schizophrenia, schizotypal and delusional disorders (ICD-10: F20-F29) and affective disorders (ICD-10: F30-F34). In both of these categories of mental disorders an upward trend in the incidence in the study population of economically active people was not observed. Affective disorders, schizophrenia, schizotypal and delusional disorders constitute the least common mental disorders in the study population.

The study group comprised economically active individuals referred by medical doctors to psychotherapy due to neurotic symptoms of depression. Individuals with severe symptoms of depression or schizophrenia are not referred to treatment in this type of medical facility. Individuals medically diagnosed with F20-F29, F30-F34 were not as numerous in the study group as individuals with neuroses and personality disorders, since acute schizophrenia, schizotypal and delusional disorders (ICD-10: F20-F29) and affective disorders (ICD-10: F30-F34) oftentimes require pharmacotherapy, hospitalization and other forms of treatment than those offered at the facilities where the study was conducted.

\section{Distribution of the incidence}

\section{of types of mental disorders by sex}

In order to answer the third research question, statistical significance was measured for the differences between the economically active female and male study participants with regard to the types of mental disorders they were medically diagnosed with, categorized as 'neurotic, stress-related and somatoform disorders' (anxiety disorders, phobias, depressive disorders and mixed anxiety and depressive disorders, stress-related disorders, somatoform disorders, personality disorders, eating disorders). Due to the marginal incidence of affective disorders, schizophrenia, schizotypal and delusional disorders in the study group, further statistical analyses of these variables were abandoned. The results showed the above data to be not sufficiently representative and, concurrently, implied the necessity to explore the subject of the incidence of affective disorders and schizophrenia, schizotypal and delusional disorders in the working population in further research on a different population. The data that describes the study results pertaining to the differences between the female and male participants with respect to neuroses, personality disorders and eating disorders are presented in the Figure 2-4.

The analysis of mean values of the incidence of neuroses, somatoform disorders and stress-related disorders proved a substantial increase in the incidence of this group of disorders in 2016 among economically active female participants. In the case of economically active male participants, the increase in the incidence of neuroses in 2016 is also notable as compared to the years 2014-2015, yet significantly lower than in the group of working female participants (Figure 2). The analysis of the results presented in the Figure $3 \mathrm{im}$ plies significant differences between economically active women and men with regard to the growing incidence of medically diagnosed personality disorders in 2014-2016.

The analysis of the incidence of personality disorders in the study population (without the distinction between the types of these disorders) shows that economically active female participants are significantly different in this regard from economically active male participants. A significant growth in the incidence of the discussed mental disorders was noted in 2014-2016 both for women and men. The highest incidence of personality disorders in both sexes occurred in 2016. However, the number of women diagnosed with personality disorders is significantly higher than that diagnosed for men. 


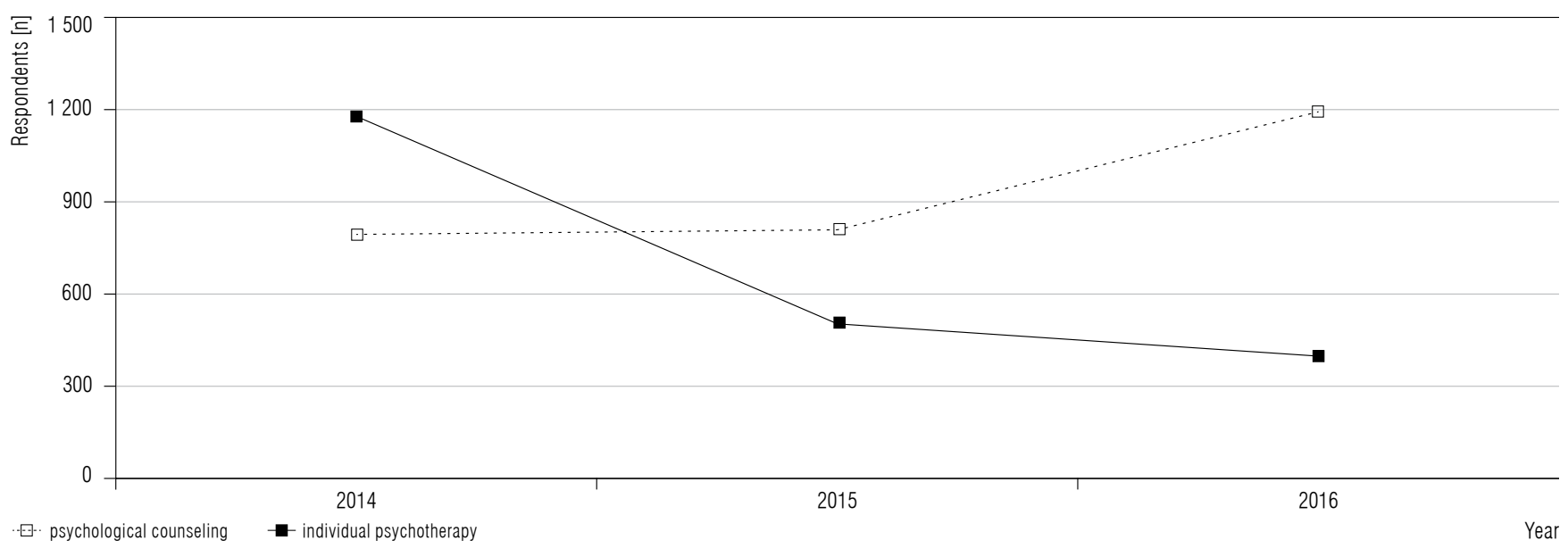

Statistically significant differences using a $\mathrm{Chi}^{2}$ test $(\mathrm{p}<0.05)$.

Fig. 6. Seeking psychological counselling or individual psychotherapy sessions due to mental disorders among the economically active respondents $(\mathrm{N}=1578)$ in Poland, 2014-2016

As for the research data provided in the Figure 4, interpretative analyses were hindered by the small number of the male participants diagnosed with eating disorders, which was insufficient for comparison with the number estimated for the female participants with eating disorders. On the other hand, the result is probably consistent with epidemiological data, since disorders of this sort affect mainly adolescent girls and young women. In order to provide a full picture, the study was complemented with an attempt to describe the study group with regard to the distribution of the age at which the economically active individuals of both sexes first sought treatment due to symptoms of mental disorders.

\section{Percentage distribution}

of the economically active individuals by age at first treatment

The Figure 5 presents the study results specifying the age at which most of the participants first sought medical help due to mental disorders experienced when fulfilling their occupational tasks.

The result analysis confirms that the range of the age at first treatment due to symptoms of various mental disorders (related exclusively to emotional disorders and not to psychoses) was very broad and did not change in 2014-2016. Both men and women undergoing treatment in the specified timeframe sought treatment due to symptoms of various mental disorders at the age of 30-64 years old. Apart from the age of first treatment due to mental disorders, the question whether economically active people resorted to counselling psychology services at the time of treatment is particularly interesting.
The data provided in the Figure 6 shows the frequency of using counselling psychology services and individual therapy sessions by the participants in 2014-2016 due to experienced emotional difficulties and reported mental disorders.

The analysis of the study results pertaining to the distribution of the frequency of provided counselling psychology services and individual therapy sessions due to mental disorders experienced by the participants showed in 2016 a significantly lower interest in individual therapy in contrast to a significantly higher frequency of counselling psychology services received in 2016.

\section{DISCUSSION}

\section{Incidence of mental disorders in the working Polish population}

As mentioned above, the number of economically active individuals diagnosed with neurotic, stress-related and somatoform disorders has significantly increased over the last 3 years, which has been the highest growth observed in the entire study population. Moreover, the growth rate in this group of mental disorders among the participants was significantly higher in 2016 as compared to the incidence of the discussed category of disorders in the years 2014 and 2015.

A question arises whether the increased incidence in this category of mental disorders (mainly neuroses and personality disorders) resulted from an improved awareness of the need to ensure good mental health and a better access to help in a situation of emotional crises appearing together with psychopathological symptoms 
of neuroses, personality disorders or other type of disorders? Alternatively, does the cause of that high growth in the incidence of neuroses (including anxiety disorders, mixed anxiety and depressive disorders, stressrelated disorders and somatoform disorders) lie in the higher severity of this type of disorders throughout the Polish population, as supported by statistical data [2-5]?

The significantly higher incidence of neuroses in the study population compared to other categories of mental disorders covered in the study (personality disorders, eating disorders and others) results from the fact that the category of neuroses is the broadest, as it involves a whole range of medical diagnoses, from phobias (F40), anxiety disorders and mixed anxiety and depressive disorders (F41, F42) to stress-related disorders (F43) and somatoform disorders (F44, F45).

The incidence of neurotic, stress-related and somatoform disorders presented in the Figure 1 covers psychopathological symptoms that mostly refer to an individual's daily emotional experiences and emotional crises. Neurotic and stress-related disorders (which are prevalent in the study population) usually involve highly severe anxiety or depressive symptomatology or psychogenic somatic symptoms. For that very reason, an economically active person may decide to seek medical help to reduce the symptoms by means of pharmacological treatment. Hence, the study findings are not surprising. It is likely that the incidence of various emotional disorders grows with the working population's awareness of the importance of mental health. Hence, one may ask to what extent does the level of mental and physical discomfort experienced by the growing number of people suffering from neuroses and personality disorders substantially hinder professional work and/or private functioning on daily basis, at times even making one unable to function, resulting in a need to resort to social isolation, sickness absenteeism or even labor market exit decisions?

In conclusion, the research data presented in the Table 1 and the Figure 1 suggests that the mental condition in the working population deteriorated, while the incidence of the diagnosed mental disorders significantly increased in 2014-2016, particularly those pertaining to the emotional structure of personality and mental difficulties experienced by the so-called working-age people.

A question arises as to what extent the above depicted phenomenon of the growing incidence of mental disorders is related in the discussed population to occupational stress emerging in work situation and in the working environment [25-27]. The person-environment fit model proposed by Leiter and Maslach suggests that apart from psychosocial factors and workload-related factors, occupation stress forms also due to emotional states experienced by the worker (anxiety, insecurity, depression, aggression) induced by a subjective feeling of a threat in work situation and the working environment [28-30]. Oftentimes does chronic stress serve as the source of neuroses, psychosomatic disorders with a negative effect on the worker, his or her co-workers and family [28]. The increase in the incidence of mental disorders in 2014-2016 shown in the economically active study population may support a general downward trend in the quality of mental health in the Polish population and an upward trend in the incidence of non-psychotic disorders (neuroses, depressive disorders and personality disorders), as indicated in the EZOP project [2].

When analyzing the study results one ought to consider that the study group comprised working individuals who sought medical help at a facility in a metropolitan area due to various mental disorders they experienced while living in nearby cities, towns and villages. The study does not differentiate the participants with regard to the place of residence for several reasons. The first reason pertains to the absence of such details in the medical records of the study participants. Some participants lived in villages near large cities, others were spending much time in large cities due to the place of work or studying, though they were residents of rural areas. This limiting aspect of the study requires further examination. However, the course of neuroses and other mental disorders is not usually associated with the place of residence. The mental disorders presented in this article result from specific inner conflicts, the specificity of emotional development and coping with stress, which is largely dependent on personal resources and results rather from autobiographical experiences or biological conditions. Symptoms of mental disorders are to a lesser extent associated with a direct impact of rural or urban environment (particularly in the case of personality disorders and neuroses) and are affected rather by personality factors.

The result analysis shows that the working population comprises people of various age. As indicated by the mean age of women and men who reported various types of mental disorders, which was provided in the description of the study group, the mean age of the female participants (35.4 years old) is slightly lower than that of the male participants (39.4 years old). 
Here, it should be noted that the research data provides information on the mean age at which the participants sought medical and psychological help due to the experienced disorders and does not determine the onset of the experienced disorders reported by the participants. The mean age declared in the interview at which the participants first sought help due to the experienced mental disorders may be interpreted as above average for the incidence of neuroses and personality traits. The determined age does not indicate the age at which the symptoms were first experienced. The participants might have been experiencing various emotional difficulties and neurotic symptoms earlier in life without realizing the disorder and the need for treatment or being ashamed of revealing these experiences to others. In Poland, seeking psychiatric help is still poorly grounded and oftentimes involves shame and fear of admitting the need for contacting a psychiatrist due to related symptoms that may be suffered for many years.

The above result suggests that economically active women seek medical and/or psychological help sooner than men when experiencing psychopathological symptoms of disorders medically diagnosed as neuroses (anxiety disorders, mixed anxiety and depressive disorders, somatoform disorders, stress-related disorders, personality disorders, eating disorders, etc.).

This finding may also be explained by the fact that economically active men might be less likely to admit to experiencing physical ailments and in order to seek medical help, they need more time to reach the level of frustration due to psychopathological symptoms indicative of disorders than economically active women. Such decision is probably dictated by social pressure (exerted by both familial and working environment due to the increased inability to work). Alternatively, the significantly lower number of economically active older men seeking medical and psychological help due to various mental disorders (both neuroses and personality disorders) may be explained by the hypothesis stating that, as evidenced by statistical data, men are more likely to seek to relieve emotional tension by engaging in psychoactive substance use (alcohol) than women are.

When analyzing and interpreting the study results one should take into consideration study limitations resulting from the fact that the data was obtained from a single facility and thus the results drawn from this data cannot be applied to the population of professionally active Poles. However, the broad research material obtained in the course of the 3-year study may be con- sidered an important empirical source of a documented knowledge on mental disorders in a vast number of working individuals residing in a province in southern Poland. Though this constitutes a study limitation (sampling of participants from a single location only), the large size of the study group sampled over a 3-year period with the very same diagnostic and treatment conditions, and with the same team of diagnosticians involved (identical diagnostic criteria and treatment procedure) are factors that support the reliability of the obtained results.

The study is limited to a considerable extent, resulting in the lack of measurement and analyses of the detailed distribution of specific types of anxiety disorders and, separately, mood disorders and types of personality disorders. The said measurement was not examined as the mentioned data was inaccessible at the time of the study. Hence, the above objective ought to be pursued in further research. Once determined, this variability in the distribution of anxiety disorders, depressive disorders and personality disorders in the working population would significantly broaden the source material that is presently rare in Polish scientific publications pertaining to the field of industrial and organizational psychology.

\section{Distribution of mental disorders in the economically active participants}

The analysis of the study results supports the prevailing position of working women over working men with regard to the number of cases reported in 2014-2016 due to various mental disorders in order to seek medical and/or psychological help. Neuroses proved to be the most prevalent of the diagnosed disorders, followed by personality disorders, which were substantially less severe compared to the former. The obtained data may explain the upward trend in the incidence of anxiety and depressive disorders in the Polish population [5], as mentioned in the Introduction of this paper

It is likely that these findings suggest a tendency that brings to light the higher motivation to seek treatment among women rather than in men. This pertains to the incidence of each of the verified types of mental disorders and may be explained to a degree by the fact that the study groups of both sexes did not involve any individuals with diagnosed psychoactive substance use disorder (alcohol and drugs) mentioned in the records. This group of diagnosed persons is not subject to treatment in the facility where the study was conducted. Persons diagnosed with psychoactive substance use disorder 
are referred to a substance abuse treatment clinic and an alcohol and drug addiction center. For that very reason, the study results are also limited with regard to the interpretation of the source material. Men diagnosed with personality disorders oftentimes tend to be prone to addiction, which suggests that they can seek and receive treatment in addiction centers. Such individuals were not included into the study group, which makes the study results not applicable to the entire population. Importantly, this matter requires further research with regard to this type of disorders, which could be pursued, e.g., by conducting future studies at alcohol and drug addiction centers and outpatient clinics.

In the case of schizophrenia, schizotypal and delusional disorders (F20-F29), individuals aged 30 years old and older often remain on disability pensions, and for that very reason they were not involved in the study group at the facility where the research was conducted. This might limit the interpretation of the obtained results on the professional activity of people diagnosed with F20-F29. Such data was not verified in this study. In this respect, a more detailed approach in further research would contribute to a broader source material on the subject of mental disorders in the working population that the one analyzed in this paper.

The findings may also be affected by the fact that the number of women who sought medical and psychological help in 2014-2016 was considerably higher that the number of men (Table 1). The analysis of the findings suggests that the prevalent age of first treatment sought by the study participants due to mental disorders is a wide age bracket of 30-64 years old. This bracket constitutes a development period that covers professional activity in a broad sense and ends with the retirement period. A similar upward trend prevails in the years 2014-2016. The second age group to seek treatment due to mental disorders is the age bracket 19-29 years old, i.e., the period of life referred to as young adulthood when professional work is undertaken for the first time. This trend prevailed in the years 2014-2016.

Importantly, the age at first treatment in this timeframe is similar, i.e., 30-64 years old. It is a broad bracket that includes the so-called working-age individuals and ought to be examined in more depth in further research, e.g., by taking into consideration a more differentiated age brackets such as, 30-50 years old (developing professional activity), 55-64 years old (stable professional activity). The differentiation suggested above might be justified by the specificity of professional work in the proposed age brackets. The study results show that the participants used counselling psychology services substantially more often than individual psychotherapy due to mental disorders in 2016. This data may suggest, first of all, a poorer access to individual psychotherapy services rendered under the contract with the National Health Fund in comparison to counselling psychology services. However, the poorer access to psychotherapy and, thus, the better access to counselling psychology services, together with emotional crises and neuroses (Table 1) prevailing in the study population may imply an enhanced need for seeking psychological help and counselling psychology services.

Given the prevalent incidence of neuroses in this group of economically active people it should be noted that the number of counselling psychology services and individual therapy services rendered in the years 2014-2016 clearly exceeds the number of participants diagnosed in the specified period. This finding suggests that economically active study participants used psychological help multiple times and, moreover, it may imply a growing demand in this field. Considering the development of occupational stress, emotional crises and various mental disorders experienced by working-age persons, it is worth to emphasize the importance of psychological help in mental health prevention and the need for providing psychological assistance to economically active individuals in various stressful situations they encounter. In reference to the statistical data obtained from EZOP report of 2012 [2] and to conclude the study results that confirm the upward trend in both the incidence of mental disorders and the number of counselling psychology services received by the study participants in 2014-2016, it is worth to note the growing need for supporting the mental condition of the working Polish population due to a significant tendency to develop mental disorders by this group.

\section{CONCLUSIONS}

The following research conclusions may be drawn from the obtained findings:

1. The presented study results pertaining to the distribution of diagnosed mental disorders should be treated with caution, as they might be associated with the specificity of the study group that comprised working Poles from a selected (yet vast) area in southern Poland. Nevertheless, the large number of participants who took part in the 3-year study allows the obtained findings to be considered a sig- 
nificant source material on the current mental condition of a large group of working Poles.

2. In the years 2014-2016, a significant growth in the incidence of various types of mental disorders was observed in the study population of economically active men and women residing in southern Poland. The prevalent upward trend pertained to the incidence of neurotic disorders diagnosed due to ICD-10 as a spectrum of neurotic, stress-related and somatoform disorders (including anxiety disorders, mixed anxiety and depressive disorders), as well as personality disorders.

3. A significant difference was observed between the incidence of mental disorders reported by economically active men and women. In the analyzed period of 2014-2016 the incidence was higher for all types of mental disorders in economically active women than in economically active men.

4. In 2014-2016, symptoms of medically diagnosed emotional disorders classified as neurotic, stress-related and somatoform disorders were prevalent in economically active women. Here, a question arises as to what extent do men fail to associate the experienced neurotic symptoms with a disease or seek to solve their emotional crises related to their life situation and occupational stress in other ways, e.g., by using psychoactive substances (including alcohol) more frequently, thus being referred to Substance Abuse Treatment Clinics? This hypothesis may be supported by modern epidemiology of substance use disorders. The absence of this group of disorders in the analysis constitutes a limitation in the study. The said aspect ought to be included as an objective in future research.

5. The demand for counselling psychology services increased significantly in 2014-2016 in the working population due to various types of mental disorders. The research data implies that counselling psychology services need to be included into mental health prevention as a means applied in the treatment of economically active individuals.

\section{REFERENCES}

1. Cybula-Fujiwara A, Merecz-Kot D, Walusiak-Skorupa J, Marcinkiewicz A, Wiszniewska M. [Employees with mental illness - Possibilities and barriers in professional activity]. Med Pr. 2015;66(1):57-69, https://doi.org/10.13075/ mp.5893.00173.

2. Kiejna A, Adamowski T, Piotrowski P, Moskalewicz J, Wojtyniak B, Świątkiewicz G, et al. [Epidemiology of psychi- atric disorders and the accessibility of psychiatric services. EZOP - Poland - Research methodology]. Psychiatr Pol. 2015;49(1):5-13, https://doi.org/10.12740/PP/30810.

3. Wciórka B, Wciórka J. [Mentally ill individuals in the society: A research report]. Warszawa: Centre for Public Opinion Research; 2008.

4. Kaszyński H, Cechnicki A. [Polish employers and employment of the mentally ill]. Psychiatr Pol. 2011;45(1):45-60.

5. Kaszyński H. [The mentally ill as a group of beneficiaries of non-governmental organizations rendering services on the labor market]. Warszawa: Polish-American Freedom Foundation; 2006.

6. Wittchen HU, Jacobi F, Rehm J, Gustavsson A, Svensson M, Jönsson B, et al. The size and burden of mental disorders and other disorders of the brain in Europe 2010. Eur Neuropsychopharmacol. 2011;21(9):655-79, https://doi.org/ 10.1016/j.euroneuro.2011.07.018.

7. [Ministry of Family, Labour and Social Policy. Directive 89/391/EEC on the introduction of measures to encourage improvements in the safety and health of workers at work [Internet]. Warszawa: The Ministry; 2017] [cited 2006 Feb 26]. Available from: https://www.mpips.gov. $\mathrm{pl} / \mathrm{gfx} / \mathrm{mpips} /$ userfiles/File/warunkipracy/dyrektywy\%20 ewg/Dyrektywa\%20_89.391.pdf. Polish.

8. Mallick K, Reeves RJ, Dellario DJ. Barriers to community integration for people with severe and persistent disabilities. Psychiatr Rehabil J. 1998;22(2):175-80, https://doi. org/10.1037/h0095250.

9. Bond GR, Resnick SG, Drake RE, Xie H, McHugo GJ, Bebout RR. Does competitive employment improve nonvocational outcomes for people with severe mental illness? J Consult Clin Psychol. 2001;69(3):489-501, https://doi. org/10.1037/0022-006X.69.3.489.

10. Koletsi M, Niersman A, van Busschbach JT, Catty J, Becker T, Burns T, et al. Working with mental health problems: Clients' experiences of IPS, vocational rehabilitation and employment. Soc Psychiatry Psychiatr Epidemiol. 2009; 44(11):961-70, https://doi.org/10.1007/s00127-009-0017-5.

11. Bio DS, Gattaz WF. Vocational rehabilitation improves cognition and negative symptoms in schizophrenia. Schizophr Res. 2011;126(1-3):265-9, https://doi.org/10.1016/j.schres. 2010.08.003

12. Kiviniemi M, Suvisaari J, Pirkola S, Laksy K, Hakkinen U, Isohanni $\mathrm{M}$, et al. Five-year follow-up study of disability pension rates in first-onset schizophrenia with special focus on regional differences and mortality. Gen Hosp Psychiatry. 2011;33(5):509-17, https://doi.org/10.1016/j.genhosppsych.2011.05.017.

13. McGurk SR, Meltzer HY. The role of cognition in vocational functioning in schizophrenia. Schizophr Res. 
2000;45(3):175-84, https://doi.org/10.1016/S0920-9964(99) 00198-X.

14. Gilmour H, Patten SB. Depression and work impairment. Health Rep. 2007;18(1):9-22.

15. Lerner D, Adler DA, Chang H, Lapitsky L, Hood MY, Perissinotto C, et al. Unemployment, job retention, and productivity loss among employees with depression. Psychiatr Serv. 2004;55(12):1371-8, https://doi.org/10.1176/appi.ps. 55.12 .1371 .

16. Burton WN, Pransky G, Conti DJ, Chen C-Y, Edington DW. The association of medical conditions and presenteeism. J Occup Environ Med. 2004;46(Suppl 6):38-45, https://doi.org/10.1097/01.jom.0000126687.49652.44.

17. Backenstrass M, Frank A, Joest K, Hingmann S, Mundt C, Kronmüller K-T. A comparative study of nonspecific depressive symptoms and minor depression regarding functional impairment and associated characteristics in primary care. Compr Psychiatry. 2006;47(1):35-41, https://doi. org/10.1016/j.comppsych.2005.04.007.

18. Beck A, Crain AL, Solberg LI, Unützer J, Glasgow RE, Maciosek MV, et al. Severity of depression and magnitude of productivity loss. Ann Fam Med. 2011;9(4):305-11, https:// doi.org/10.1370/afm.1260.

19. Wang PS, Simon GE, Avorn J, Azocar F, Ludman EJ, McCulloch J, et al. Telephone screening, outreach, and care management for depressed workers and impact on clinical and work productivity outcomes: A randomized controlled trial. JAMA. 2007;298(12):1401-11, https://doi.org/10.10 01/jama.298.12.1401.

20. Act on the protection of mental health. J Laws 2017, No. 111, item 53.
21. Ministry of Health. Information on the actions implemented under the National Mental Health Protection Program in 2014. Warszawa: The Ministry; 2015.

22. Ministry of Health. National Strategic Framework. Policy Paper for the protection of health for the period 20142020. Warszawa: The Ministry; 2015.

23. Lazarus RS, Folkman S. Stress, appraisal and coping. New York: Springer; 1984.

24. Hobfoll SE. Conservation of resources: A new attempt at conceptualizing stress. Am Psychologist. 1989;44(3): 513-24, https://doi.org/10.1037/0003-066X.44.3.513.

25. Leiter PM. Areas of Worklife Survey Manual. Wolfville: Centre for Organizational Research and Development, Acadia University; 2006.

26. Maslach C, Leiter MP. Early predictors of job burnout and engagement. J App Psychol. 2008;93(3):498-512, https:// doi.org/10.1037/0021-9010.93.3.498.

27. Petterson IL, Arnetz BB. Psychosocial stressors and wellbeing in health care workers. The impact of an intervention program. Soc Sci Med. 1998;47:1763-72, https://doi. org/10.1016/S0277-9536(98)00245-7.

28. Appelbaum E, Bailey T, Berg P, Kalleberg A. Organizations and the intersection of work and family. A comparative perspective. In: Ackroyd S, Batt R, Thompson P, Tolber PS, editors. The Oxford handbook of work \& organization. Oxford: Oxford University Press; 2005. p. 37-56.

29. World Health Organization. International statistical classification of diseases and related health problems ICD10 10th revision. Geneva: The Organization; 2010.

This work is available in Open Access model and licensed under a Creative Commons Attribution-NonCommercial 3.0 Poland License / Ten utwór jest dostępny w modelu open access na licencji Creative Commons Uznanie autorstwa - Użycie niekomercyjne 3.0 Polska - http://creativecommons.org/ licenses/by-nc/3.0/pl/deed.en. 